\title{
A Variance Formula Related to a Quantum Conductance Problem
}

\author{
TIEFENG JiAng ${ }^{1}$
}

\begin{abstract}
Let $t$ be a block of an Haar-invariant orthogonal $(\beta=1)$, unitary $(\beta=2)$ or symplectic $(\beta=4)$ matrix from the classical compact groups $O(n), U(n)$ or $S p(n)$, respectively. We obtain a close form for $\operatorname{Var}\left(\operatorname{tr}\left(t^{*} t\right)\right)$. The case for $\beta=2$ is related to a quantum conductance problem, and our formula recovers a result obtained by several authors. Moreover, our result shows that the variance has a limit $(8 \beta)^{-1}$ for $\beta=1,2$ and 4 as the sizes of $t$ go to infinity in a special way. Although $t$ in our formulation comes from a block of an Haar-invariant matrix from the classical compact groups, the above limit is consistent with a formula by Beenakker, where $t$ is a block of a circular ensemble.
\end{abstract}

\section{Introduction}

We first describe a conductance problem revelent to a formula that will be obtained in this paper. Suppose some electron fluxes travel through a wire with left and right edges. To be precise, let the $n$-component vector $\vec{I}$ and $m$-component vector $\overrightarrow{I^{\prime}}$ stand for the complex amplitudes of the available plane wave states traveling into the left and right sides of the wire, respectively. Let $\vec{O}$ and $\overrightarrow{O^{\prime}}$ be the $m$-component and $n$-component vectors for the same states traveling out of the left and right sides of the wire. The so-called scattering matrix $S$ connects the two pair of quantities in the following way:

$$
S\left(\begin{array}{l}
\vec{I} \\
\overrightarrow{I^{\prime}}
\end{array}\right)=\left(\begin{array}{l}
\vec{O} \\
\overrightarrow{O^{\prime}}
\end{array}\right)
$$

For convenience of discussion, write

$$
S=\left(\begin{array}{cc}
r_{n \times n} & t_{n \times m} \\
\tilde{t}_{m \times n} & \tilde{r}_{m \times m}
\end{array}\right),
$$

where $t$ and $\tilde{t}$ are transmission matrices, and $r$ and $\tilde{r}$ are reflection matrices. Because of flux conservation, one has to have $|\vec{I}|^{2}+\left|\overrightarrow{I^{\prime}}\right|^{2}=|\vec{O}|^{2}+\left|\overrightarrow{O^{\prime}}\right|^{2}$. This requires that $S$ has to be unitary. Since $S$ is unknown, it is reasonable to assume that $S$ is random and unitary. There are three cases in the system: a) there is no time reversal symmetry; b) there is a time reversal symmetry with $T^{2}=1 ; \mathrm{c}$ ) there is a time reversal symmetry with $T^{2}=-1$. By the above statement, the conductance $G$ is described through $t$ :

$$
G / G_{0}=\operatorname{tr}\left(t^{*} t\right)=\operatorname{tr}\left(t t^{*}\right),
$$

\footnotetext{
${ }^{1}$ Supported in part by NSF \#DMS-0449365,

School of Statistics, University of Minnesota, 224 Church Street, MN55455, tjiang@stat.umn.edu.
} 
where $(1 / 2) G_{0}=e^{2} / h$ is the fundamental unit of conductance. Relating $S$ to an evolution operator or a Hamiltonian, one may think that $S^{*}=S$. Assuming that $S$ is a random matrix from the Circular Orthogonal Ensemble (COE, $\beta=1$ ), the Circular Unitary Ensemble (CUE, $\beta=2$ ) or the Circular Symplectic Ensemble (CSE, $\beta=4)$, it is shown in $[2,4,18,24,34]$ that

$$
\operatorname{Var}\left(G / G_{0}\right)=\frac{\gamma m(m-1+\gamma) n(n-1+\gamma)}{(N-2+\gamma)(N-1+\gamma)^{2}(N-1+2 \gamma)}
$$

where $N=m+n$ and $\gamma=2 / \beta$ for $\beta=1,2,4$. For definitions and properties of the three ensembles, one can see chapter 9 from [28]. A formula of $\operatorname{Etr}\left(\left(t t^{*}\right)^{n}\right)$ for $\beta=2$ and any $n \geq 1$ is given in [31]. It is not difficult to see that (1.4) implies the following Beenakker's formula ([3])

$$
\lim _{n \rightarrow \infty} \operatorname{Var}\left(G / G_{0}\right)=\frac{1}{8 \beta}
$$

with $\alpha:=n-m+1-\beta^{-1}$ being fixed.

Note that an $n \times n$ random matrix from the CUE and an Haar-invariant unitary matrix with the same size have the same probability distribution. An $n \times n$ Haar-invariant unitary matrix generates the Haar probability measure on the unitary group $U(n)$, which is one of the three classical compact groups: the orthogonal group $O(n)(\beta=1)$, the unitary group $U(n)(\beta=2)$ and the symplectic group $S p(n)(\beta=4)$. Now, assuming $S$ in (1.2) is not in general from a Circular Ensemble, but following the three Haar probability measures, we next compute the corresponding variance of $G / G_{0}$ as in (1.3).

THEOREM 1 Assuming $S$ in (1.2) is a random matrix which is Haar orthogonal from $O(n)(\beta=$ 1), Haar unitary from $U(n)(\beta=2)$, or Haar symplectic from $\operatorname{Sp}(n)(\beta=4)$, we then have

$$
\operatorname{Var}\left(G / G_{0}\right)=\frac{\gamma m^{2} n^{2}}{(N-1) N^{2}(N+\gamma)}
$$

for any $m \geq 1, n \geq 1$ and $\beta=1,2$ and 4 , where $N=m+n$ and $\gamma=2 / \beta$.

Evidently, the formulas in (1.4) and (1.6) are the same for $\beta=2$. This is because, as mentioned earlier, a random matrix from the CUE and an Haar-invariant unitary matrix from $U(n)$ of the same size have the same distribution. Although the two formulas in (1.4) and (1.6) are generally different for $\beta=1$ and $\beta=4$, fixing $\alpha:=n-m+1-\beta^{-1}$, the limits of the two are identical. In fact, we have the following corollary from Theorem 1 .

COROLlaRY 1.1 Under the assumptions in Theorem 1, we have that $\lim _{m \rightarrow \infty} \operatorname{Var}\left(G / G_{0}\right)=$ $1 /(8 \beta)$ for $\beta=1,2$ and 4 , provided $\alpha:=n-m+1-\beta^{-1}$ is a constant.

As far as the mean values of $G / G_{0}$, we know

$$
E\left(G / G_{0}\right)= \begin{cases}\frac{m n}{N-1+\gamma}, & \text { if } S \text { is from } \operatorname{COE}(\beta=1), \operatorname{CUE}(\beta=2) \text { or } \operatorname{CSE}(\beta=4) \\ \frac{m n}{N}, & \text { if } S \text { is Haar-invariant on } O(n), U(n) \text { or } S p(n)\end{cases}
$$


where $\gamma=2 / \beta$. The first identity in (1.7) is from (8) in [34]; the second is from the Haar invariant property, see the first assertion in (2.7). The two quantities on the right above are identical for $\beta=2$, and very close to each other for $\beta=1$ and 4 .

Remark 1. When $S$ in (1.2) is an Haar-invariant orthogonal, unitary or symplectic matrix from $O(n), U(n)$ or $S p(n)$, respectively, the eigenvalues of $t_{n \times m}^{*} t_{n \times m}$ forms a Jacobi ensemble for $\beta=1,2$ or 4. This connection is established recently by Collins [6]. For other results in this realm, one can see $[1,7,12,14,17,30]$.

Remark 2. It is worthwhile to mention the difference and a connection between our model behind formula (1.6) and the traditional models behind formula (1.4): our model $t$ is based on a truncation part of an Haar-invariant matrix from $O(n)(\beta=1), U(n)(\beta=2)$ or $S p(n)(\beta=4)$. The $t$ in the traditional model (see, e.g., $[2,4,5,18,24,34])$ comes from a block of the Circular Orthogonal Ensemble $(\beta=1)$, the Circular Unitary Ensemble $(\beta=2)$ or the Circular Symplectic Ensemble $(\beta=4)$. However, for $\beta=2$, the Haar-invariant matrix from $U(n)$ and the $n \times n$ Circular Unitary Ensemble have the same probability distribution, see [28].

Remark 3. The method of the proof of Theorem 1 is different than that in $[2,4,5,18,24,34]$. Our method is based on the symmetry of the entries of Haar invariant matrices; the derivations mentioned in the above literatures rely on the explicit expression of the joint density function of eigenvalues $\lambda_{1}, \cdots, \lambda_{n}$ of $r^{*} r$.

Remark 4. Now we discuss a possibility to generalize (1.6) for $\beta=1,2$ and 4 to that for any $\beta>0$. The classical random matrix models, as described by Dyson [11] (see also Dumitriu and Edelman $[10])$, are the Hermite ensembles, the Laguerre ensembles and the Jacobi ensembles. In each of the three ensembles, there are three cases: $\beta=1,2$ and 4 , which correspond to the real, the complex and the quaternion Gaussian distributions. Theorem 1 and Corollary 1.1 are based on the Jacobi ensembles for $\beta=1,2,4$.

Recently, Dumitriu and Edelman [10] construct special tri-diagonal matrices whose eigenvalues have probability density functions for all $\beta>0$ for the Hermite and the Laguerre ensembles, respectively. In particular, the density functions are the same as those for $\beta=1,2$. Subsequent work can be found in, e.g., $[8,9,13,19,26,32,35]$. A recent work of Lippert [25] constructs special matrices (similar to the fashion Dumitriu and Edelman [10] used) which generalize the Jacobi ensembles for $\beta=1,2,4$ to those for any $\beta>0$; Killip and Nenciu [20] propose certain matrix models for the circular ensembles. One can see $[27]$ and literatures therein for some similar considerations of the circular ensembles. As mentioned in Remark 2, the proof of our main results rely on the symmetry of the entries of the Haar-invariant matrices from the classical compact groups. Generalizations of (1.6) for any $\beta>0$ can be possibly worked out by using the constructions of the entries of the special matrices proposed in the above literature.

On the other hand, though (1.4) is known to hold for any $\beta>0$ (see, e.g., [34]), similar to the discussion on the generalization of (1.6) above, it would be interesting to see another derivation of (1.4) by using the construction in [20]. 


\section{Proof of the Main Result}

To prove Theorem 1, we need some background about the Haar measures on the classical compact groups.

Let $\mathbb{R}$ and $\mathbb{C}$ denote the sets of real and complex numbers, respectively. Write $\mathbb{H}=\left\{a=a_{1}+a_{2} i+\right.$ $a_{3} j+a_{4} k ; a_{1}, a_{2}, a_{3}$ and $\left.a_{4} \in \mathbb{R}\right\}$ to denote the set of real quaternions, where $i^{2}=j^{2}=k^{2}=-1$ and $i j=k, j k=i, k i=j$. For $a=a_{1}+a_{2} i+a_{3} j+a_{4} k \in \mathbb{H}$, the conjugate of $a$ is $a^{*}=a_{1}-a_{2} i-a_{3} j-a_{4} k$, and the absolute value of $a$ is $|a|=\sqrt{a^{*} a}=\sqrt{a a^{*}}=\left(a_{1}^{2}+a_{2}^{2}+a_{3}^{2}+a_{4}^{2}\right)^{1 / 2}$. Given an $n \times m$ matrix $A=\left(a_{p q}\right)$ with $a_{p q} \in \mathbb{R}, \mathbb{C}$ or $\mathbb{H}$, the conjugate of $A$ is the $m \times n$ matrix $A^{*}=\left(a_{q p}^{*}\right)$. Recall

$$
\begin{aligned}
& O(n)=\left\{A=\left(a_{p q}\right)_{n \times n}: A^{*} A=I_{n} \text { and } a_{p q} \in \mathbb{R} \text { for all } 1 \leq p, q \leq n\right\} \\
& U(n)=\left\{A=\left(a_{p q}\right)_{n \times n}: A^{*} A=I_{n} \text { and } a_{p q} \in \mathbb{C} \text { for all } 1 \leq p, q \leq n\right\} \\
& S p(n)=\left\{A=\left(a_{p q}\right)_{n \times n}: A^{*} A=I_{n} \text { and } a_{p q} \in \mathbb{H} \text { for all } 1 \leq p, q \leq n\right\}
\end{aligned}
$$

They are in order called orthogonal, unitary and symplectic (quaternionic unitary) groups, see p.111 and p.113 from [21], or p.90 and p.92 from [33]. These groups are all compact.

We will need several lemmas to prove the main result. The first one is about a understanding of the Haar measure on group $S p(n)$. The lemma is based on the Gram-Schmidt procedure on independent and identically distributed (i.i.d.) normal random vectors. This method is used to study the entries of matrices which generate the Haar measures on $O(n)$ and $U(n)$, and then is applied for investigating the properties of eigenvalues of Jacobi matrices, see $[15,16,17]$. There are other ways to generate Haar measures on the classical compact groups, see e.g., Mezzadri[29].

In the next, one will see some phrases such as " $\mu$ is the normalized Haar measure on $\operatorname{Sp}(n)$ ", it means that $\mu$ is an Haar measure on $S p(n)$ and $\mu(S p(n))=1$.

LEMMA 2.1 Let $A_{n}=\left(v_{1}, v_{2}, \cdots, v_{n}\right)$ be an $n \times n$ random matrix whose probability distribution is the normalized Haar measure on $O(n)(\beta=1), U(n)(\beta=2)$ or $\operatorname{Sp}(n)(\beta=4)$. Then $v_{1}$ has the same probability distribution as that of

$$
\begin{aligned}
& \text { (i) } \frac{1}{\left\|X_{n}^{\beta}\right\|}\left(X_{11}, \cdots, X_{n 1}\right)^{T} \text { for } \beta=1 ; \\
& \text { (ii) } \frac{1}{\left\|X_{n}^{\beta}\right\|}\left(X_{11}+i X_{12}, \cdots, X_{n 1}+i X_{n 2}\right)^{T} \text { for } \beta=2 ; \\
& \text { (iii) } \frac{1}{\left\|X_{n}^{\beta}\right\|}\left(X_{11}+i X_{12}+j X_{13}+k X_{14}, \cdots, X_{n 1}+i X_{n 2}+j X_{n 3}+k X_{n 4}\right)^{T} \text { for } \beta=4,
\end{aligned}
$$

where $X_{p q} ; 1 \leq p \leq n, 1 \leq q \leq 4$ are independent $N(0,1)$-distributed random variables and $\left\|X_{n}^{\beta}\right\|^{2}=\sum_{1 \leq p \leq n, 1 \leq q \leq \beta} X_{p q}^{2}$ for $\beta=1,2$ or 4 .

Proof. The first two cases are well-known, see, e.g., [15]. Now we prove case (iii).

Let $Y=\left(Y_{p q}\right)=\left(Y_{1}, Y_{2}, \cdots, Y_{n}\right)$ be an $n \times n$ matrix, where the $Y_{i}$ 's are $n \times 1$ column vectors. Now, assume that $Y_{p q}$ 's are independent and identically distributed (i.i.d.) with the same distribution 
as that of $X_{11}+i X_{12}+j X_{13}+k X_{14}$. Recall the Gram-Schmidt orthogonalization procedures for $Y_{i}$ 's in the real and complex cases. For the quaternion case, set

$$
\begin{aligned}
& w_{1}=Y_{1} \text { and } e_{1}=\frac{w_{1}}{\left\|w_{1}\right\|} ; \\
& w_{p}=Y_{p}-\sum_{q=1}^{p-1} e_{q} \cdot\left(e_{q}^{*} Y_{p}\right) \text { and } e_{p}=\frac{w_{p}}{\left\|w_{p}\right\|} \text { for } 2 \leq p \leq n,
\end{aligned}
$$

where $\left\|w_{i}\right\|^{2}=w_{i}^{*} w_{i}$ for all $i$. The positions of $e_{q}$ and $e_{q}^{*} Y_{p}$ in the product $e_{q} \cdot\left(e_{q}^{*} Y_{p}\right)$ is vital since the quaternions are not commutative. It is understood as the product of an $n \times 1$ matrix and a $1 \times 1$ matrix. Using the property $(A B)^{*}=B^{*} A^{*}$, one obtains that $e_{p}^{*} e_{q}=w_{p}^{*} e_{q} /\left\|w_{p}\right\|=0$ for any $1 \leq q<p \leq n$. Thus, $\Gamma(Y):=\left(e_{1}, e_{2}, \cdots, e_{n}\right) \in S p(n)$. Now, take $S \in S p(n)$, define $\tilde{Y}=S Y$, and $\tilde{Y}_{p}, \tilde{w}_{p}$ and $\tilde{e}_{p}$ accordingly. First, we see that $\tilde{e}_{1}=S e_{1}$. Given $2 \leq p \leq n$, suppose $\tilde{e}_{q}=S e_{q}$ for all $1 \leq q \leq p-1$. Now, using the fact $S^{*} S=S S^{*}=I$ to have that

$$
\begin{aligned}
\tilde{w}_{p} & =\tilde{Y}_{p}-\sum_{q=1}^{p-1} \tilde{e}_{q} \cdot\left(\tilde{e}_{q}^{*} \tilde{Y}_{p}\right) \\
& =S Y_{p}-\sum_{q=1}^{p-1}\left(S e_{q}\right) \cdot\left(\left(S e_{q}\right)^{*}\left(S Y_{p}\right)=S w_{p} .\right.
\end{aligned}
$$

Thus, $\tilde{e}_{p}=S e_{p}$. By induction, we know that $\Gamma(S Y)=S \cdot \Gamma(Y)$ for any $S \in S p(n)$. Since all the entries of $Y$ are i.i.d., by the invariance, we know that $S Y$ and $Y$ have the same probability distribution, see, e.g. [36] or Theorem 1 from [23]. Therefore, $\Gamma(Y)$ and $S \cdot \Gamma(Y)$ have the same distribution for any $S \in S p(n)$. Note that $S p(n)$ is compact, the left and right Haar measures are the same (up to a positive scaler), thus $\Gamma(Y)$ generates the normalized Haar measure on $S p(n)$. The desired conclusion follows from (2.2).

Suppose $S$ is an Haar-invariant orthogonal, unitary or symplectic random matrix from $O(n), U(n)$ or $S p(n)$, respectively. We will use the following properties of $S$ from time to time in our proofs:

Exchanging any two rows or two columns of $S$, the new matrix has the same distribution as that of $S$;

The distributions of $S$ and $S^{T}$ are identical.

LEMMA 2.2 Let $r_{n \times n}=\left(r_{i j}\right)_{n \times n}$ be as in (1.2). Set $\sigma_{i j}=\left|r_{i j}\right|$ for $1 \leq i, j \leq n$, and $W_{n}=$ $\operatorname{tr}\left(r_{n \times n}^{*} r_{n \times n}\right)$. Under the conditions of Theorem 1, we have that

$$
\operatorname{Var}\left(W_{n}\right)=n^{2} \operatorname{Var}\left(\sigma_{11}^{2}\right)+2 n\left(n^{2}-n\right) \operatorname{Cov}\left(\sigma_{11}^{2}, \sigma_{21}^{2}\right)+\left(n^{2}-n\right)^{2} \operatorname{Cov}\left(\sigma_{11}^{2}, \sigma_{22}^{2}\right)
$$

for $\beta=1,2$ and 4 . 
Proof. Write $W_{n}=\sum_{1 \leq i, j \leq n} \sigma_{i j}^{2}$. Then

$$
\begin{aligned}
\operatorname{Var}\left(W_{n}\right)= & \operatorname{Cov}\left(\sum_{1 \leq i, j \leq n} \sigma_{i j}^{2}, \sum_{1 \leq i, j \leq n} \sigma_{i j}^{2}\right) \\
= & \sum_{1 \leq i, j \leq n} \operatorname{Cov}\left(\sigma_{i j}^{2}, \sigma_{i j}^{2}\right)+\sum_{i=1}^{n} \sum_{1 \leq j \neq k \leq n} \operatorname{Cov}\left(\sigma_{i j}^{2}, \sigma_{i k}^{2}\right)+\sum_{i=1}^{n} \sum_{1 \leq j \neq k \leq n} \operatorname{Cov}\left(\sigma_{j i}^{2}, \sigma_{k i}^{2}\right) \\
& +\sum_{1 \leq i \neq k \leq n} \sum_{1 \leq j \neq l \leq n} \operatorname{Cov}\left(\sigma_{i j}^{2}, \sigma_{k l}^{2}\right) .
\end{aligned}
$$

By (2.3) and (2.4), the first term above is equal to $n^{2} \operatorname{Var}\left(\sigma_{11}^{2}\right)$; both the second and third are identical to $n\left(n^{2}-n\right) \operatorname{Cov}\left(\sigma_{11}^{2}, \sigma_{21}^{2}\right)$; the last term is $\left(n^{2}-n\right)^{2} \operatorname{Cov}\left(\sigma_{11}^{2}, \sigma_{22}^{2}\right)$.

LEMMA 2.3 Let the conditions in Theorem 1 hold. Recall $N=m+n$. Then the following holds for $\beta=1,2$ and 4 :

(i) $\operatorname{Var}\left(\sigma_{11}^{2}\right)=-(N-1) \cdot \operatorname{Cov}\left(\sigma_{11}^{2}, \sigma_{21}^{2}\right)$;

(ii) $\operatorname{Cov}\left(\sigma_{11}^{2}, \sigma_{22}^{2}\right)=-1 /(N-1) \cdot \operatorname{Cov}\left(\sigma_{11}^{2}, \sigma_{21}^{2}\right)$.

Proof. Since $\sum_{i=1}^{N} \sigma_{i 1}^{2}=1$, we have from (2.3) that

$$
0=\operatorname{Var}\left(\sum_{i=1}^{N} \sigma_{i 1}^{2}\right)=N \cdot \operatorname{Var}\left(\sigma_{11}^{2}\right)+N(N-1) \cdot \operatorname{Cov}\left(\sigma_{11}^{2}, \sigma_{21}^{2}\right)=0 .
$$

So (i) follows. Now, by the fact $\sum_{i=1}^{N} \sigma_{i 2}^{2}=1$ and by (2.3) again,

$$
\begin{aligned}
0 & =\operatorname{Cov}\left(\sum_{i=1}^{N} \sigma_{i 1}^{2}, \sum_{i=1}^{N} \sigma_{i 2}^{2}\right) \\
& =N \cdot \operatorname{Cov}\left(\sigma_{11}^{2}, \sigma_{12}^{2}\right)+N(N-1) \cdot \operatorname{Cov}\left(\sigma_{11}^{2}, \sigma_{22}^{2}\right) .
\end{aligned}
$$

By (2.4), we know that $\operatorname{Cov}\left(\sigma_{11}^{2}, \sigma_{12}^{2}\right)=\operatorname{Cov}\left(\sigma_{11}^{2}, \sigma_{21}^{2}\right)$, assertion (ii) is obtained.

Recall notation $(2 m-1) ! !=(2 m-1)(2 m-3) \cdots 3 \cdot 1$ for any integer $m \geq 1$, and $(-1) ! !=1$ by convention.

LEMMA 2.4 Suppose $n \geq 2$ and $Z_{1}, Z_{2}, \cdots, Z_{n}$ are i.i.d. random variables with $Z_{1} \sim N(0,1)$. Define $U_{i}=Z_{i}^{2} /\left(Z_{1}^{2}+\cdots Z_{n}^{2}\right)$ for $1 \leq i \leq n$. Let $a_{1}, \cdots, a_{n}$ be non-negative integers and $a=\sum_{i=1}^{n} a_{i}$. Then

$$
E\left(U_{1}^{a_{1}} U_{2}^{a_{2}} \cdots U_{n}^{a_{n}}\right)=\frac{\prod_{i=1}^{n}\left(2 a_{i}-1\right) ! !}{\prod_{i=1}^{a}(n+2 i-2)} .
$$

Proof. It is known that $\left(U_{1}, U_{2}, \cdots, U_{n-1}\right)$ follows the Dirichlet distribution whose parameters are all equal to $1 / 2$, that is, their joint density function is

$$
f\left(u_{1}, u_{2}, \cdots, u_{n-1}\right)=\frac{\Gamma(n / 2)}{\Gamma(1 / 2)^{n}} u_{1}^{-1 / 2} \cdots u_{n-1}^{-1 / 2}\left(1-\sum_{i=1}^{n-1} u_{i}\right)^{-1 / 2}
$$


for $u_{i} \geq 0$ and $\sum_{j=1}^{n-1} u_{j} \leq 1$, see, e.g., chapter 49 from [22]. Then

$$
\begin{aligned}
& E\left(U_{1}^{a_{1}} U_{2}^{a_{2}} \cdots U_{n}^{a_{n}}\right) \\
= & \frac{\Gamma(n / 2)}{\Gamma(1 / 2)^{n}} \int \cdots \int u_{1}^{a_{1}-(1 / 2)} \cdots u_{n-1}^{a_{n-1}-(1 / 2)}\left(1-\sum_{i=1}^{n-1} u_{i}\right)^{a_{n}-(1 / 2)} d u_{1} \cdots d u_{n-1} \\
= & \frac{\Gamma(n / 2)}{\Gamma(1 / 2)^{n}} \cdot \frac{\prod_{j=1}^{n} \Gamma\left(a_{j}+(1 / 2)\right)}{\Gamma(a+(n / 2))}
\end{aligned}
$$

since the integral is the reciprocal of the normalizing constant of a Dirichlet distribution with parameters $a_{j}+1 / 2(j=1, \cdots, n)$. Now, using the fact that $\Gamma(x+1)=x \Gamma(x)$ for $x \geq 0$ to obtain

$$
\begin{aligned}
\Gamma\left(a+\frac{n}{2}\right) & =\left(a-1+\frac{n}{2}\right)\left(a-2+\frac{n}{2}\right) \cdots \frac{n}{2} \Gamma\left(\frac{n}{2}\right) \\
& =\frac{n(n+2) \cdots(n+2(a-1))}{2^{a}} \Gamma\left(\frac{n}{2}\right) .
\end{aligned}
$$

Substituting $a=a_{j}$ and $n=1$ in the above formula, we have that

$$
\prod_{j=1}^{n} \Gamma\left(a_{j}+\frac{1}{2}\right)=\frac{\Gamma(1 / 2)^{n}}{2^{a}} \prod_{j=1}^{n}\left(2 a_{j}-1\right) ! !
$$

The lemma is proved.

LEMMA 2.5 Let $Z_{1}, Z_{2}, \cdots$ be a sequence of i.i.d. random variables with $Z_{1} \sim N(0,1)$. Then

$$
\operatorname{Cov}\left(\frac{Z_{1}^{2}+\cdots+Z_{\beta}^{2}}{Z_{1}^{2}+\cdots+Z_{\beta l}^{2}}, \frac{Z_{\beta+1}^{2}+\cdots+Z_{2 \beta}^{2}}{Z_{1}^{2}+\cdots+Z_{\beta l}^{2}}\right)=-\frac{2}{l^{2}(\beta l+2)}
$$

for $\beta=1,2$ and 4 and any integer $l \geq 2$.

Proof. By Lemma 2.4,

$$
E \frac{Z_{1}^{2}}{Z_{1}^{2}+\cdots+Z_{k}^{2}}=\frac{1}{k} \text { and } E \frac{Z_{1}^{2} Z_{2}^{2}}{\left(Z_{1}^{2}+\cdots+Z_{k}^{2}\right)^{2}}=\frac{1}{k(k+2)}
$$

for all $k \geq 2$. Then, by the bilinear property of covariance, the left hand side of (2.6) is identical to

$$
\begin{aligned}
& \beta^{2} \cdot \operatorname{Cov}\left(\frac{Z_{1}^{2}}{Z_{1}^{2}+\cdots+Z_{\beta l}^{2}}, \frac{Z_{2}^{2}}{Z_{1}^{2}+\cdots+Z_{\beta l}^{2}}\right) \\
= & \beta^{2}\left\{E \frac{Z_{1}^{2} Z_{2}^{2}}{\left(Z_{1}^{2}+\cdots+Z_{\beta l}^{2}\right)^{2}}-\left(E \frac{Z_{1}^{2}}{Z_{1}^{2}+\cdots+Z_{\beta l}^{2}}\right)^{2}\right\} \\
= & \beta^{2}\left(\frac{1}{\beta l(\beta l+2)}-\frac{1}{(\beta l)^{2}}\right) .
\end{aligned}
$$

The conclusion follows.

Proof of Theorem 1. Since the length of each row and that of each column of $S$ in (1.2) is one, we have that

$$
\operatorname{tr}\left(r_{n \times n}^{*} r_{n \times n}\right)+\operatorname{tr}\left(t_{n \times m}^{*} t_{n \times m}\right)=n \text { and } \operatorname{tr}\left(t_{n \times m}^{*} t_{n \times m}\right)+\operatorname{tr}\left(\tilde{r}_{m \times m}^{*} \tilde{r}_{m \times m}\right)=m .
$$


Thus, $\operatorname{Var}\left(\operatorname{tr}\left(t^{*} t\right)\right)=\operatorname{Var}\left(\operatorname{tr}\left(r^{*} r\right)\right)=\operatorname{Var}\left(\operatorname{tr}\left(\tilde{r}^{*} \tilde{r}\right)\right)$, where $t=t_{n \times m}, r=r_{n \times n}$ and $\tilde{r}=\tilde{r}_{m \times m}$. Now, reviewing (1.2), by using (2.4), we know that

$$
S=\left(\begin{array}{cc}
r_{n \times n} & t_{n \times m} \\
\tilde{t}_{m \times n} & \tilde{r}_{m \times m}
\end{array}\right) \text { and }\left(\begin{array}{cc}
\tilde{r}_{m \times m} & \tilde{t}_{m \times n} \\
t_{n \times m} & r_{n \times n}
\end{array}\right)
$$

have the same distribution. Thus, without loss of generality, assume that $n \leq m$. As in Lemma 2.2, let $W_{n}=\operatorname{tr}\left(r^{*} r\right)$. It suffices to prove that

$$
\operatorname{Var}\left(W_{n}\right)=\frac{2 \beta^{-1} m^{2} n^{2}}{(N-1) N^{2}\left(N+2 \beta^{-1}\right)}
$$

for $\beta=1,2$ and 4 . Recalling that $r=\left(r_{i j}\right)$ and $\sigma_{i j}=\left|r_{i j}\right|$, by Lemma 2.1 and (2.3),

$$
\left(\sigma_{11}^{2}, \sigma_{21}^{2}\right),\left(\sigma_{11}^{2}, \sigma_{12}^{2}\right) \text { and }\left(\frac{\sum_{i=1}^{\beta} Z_{i}^{2}}{\sum_{i=1}^{N \beta} Z_{i}^{2}}, \frac{\sum_{i=\beta+1}^{2 \beta} Z_{i}^{2}}{\sum_{i=1}^{N \beta} Z_{i}^{2}}\right)
$$

are identical in distribution, where $\left\{Z_{i} ; i=1,2, \ldots\right\}$ is a sequence of i.i.d. $N(0,1)$-distributed random variables. By Lemma 2.5,

$$
\operatorname{Cov}\left(\sigma_{11}^{2}, \sigma_{21}^{2}\right)=-\frac{2}{N^{2}(\beta N+2)} .
$$

This together with Lemmas 2.2 and 2.3 yields that

$$
\begin{aligned}
\operatorname{Var}\left(W_{n}\right) & =\left(-(N-1) n^{2}+2 n^{2}(n-1)-\frac{n^{2}(n-1)^{2}}{N-1}\right) \operatorname{Cov}\left(\sigma_{11}^{2}, \sigma_{21}^{2}\right) \\
& =-n^{2}\left(\frac{(N-1)^{2}-2(n-1)(N-1)+(n-1)^{2}}{N-1}\right) \operatorname{Cov}\left(\sigma_{11}^{2}, \sigma_{21}^{2}\right) \\
& =-n^{2} \frac{(N-n)^{2}}{N-1} \operatorname{Cov}\left(\sigma_{11}^{2}, \sigma_{21}^{2}\right) \\
& =\frac{2 m^{2} n^{2}}{(N-1) N^{2}(\beta N+2)} .
\end{aligned}
$$

The proof is complete.

Acknowledgement. The author thanks Professor Peter Forrester for a discussion on the conductance problem mentioned in this paper; thanks the anonymous referees for their careful reading of this paper and providing valuable suggestions for the revision; thanks X. Ding and D. Li for helpful discussions on the proofs of the main results in this paper.

\section{References}

[1] Anderson, T.W. (1984). An Introduction to Multivariate Statistical Analysis. John Wiley \& Sons, Second edition.

[2] Baranger, H. U. and Mello, P.A. (1994). Mesoscopic transport through chaotic cavities: A random S-matrix theory approach. Phys. Rev. Lett. 73, 142 - 145. 
[3] Beenakker, C. W. J. (1993). Universality in the random matrix theory of quantum transport. Phys. Rev. Lett. 70, 1155-1158.

[4] Beenakker, C. W. J. (1997). Random-Matrix Theory of Quantum Transport. Rev. Mod. Phys. 69, 731-808.

[5] Brody, T. A., Flores, J., French, J. B., Mello, P. A., Pandey, A., Wong, S. S. M. (1981). Random-matrix physics: spectrum and strength fluctuations. Reviews of Modern Physics 53(3), 385-479.

[6] Collins, B. (2005). Product of random projections, Jacobi ensembles and universality problems arising from free probability. Probability theory and related fields 133, 315-344.

[7] Constantine, A. G. (1963). Some non-central distribution problems in multivariate analysis. Ann. Math. Stat. 34 1270-1285.

[8] Dumitriu, I. and Edelman, A. (2006). Global spectrum fluctuations for the beta-Hermite and beta-Laguerre ensembles via matrix models. Journal of Mathematical Physics 47, 063302 .

[9] Dumitriu, I. and Edelman, A. (2005). Eigenvalues of Hermite and Laguerre Ensembles: Large Beta Asymptotics. Annales de l'Institut Henri Poincare (B), 41(6), 1083-1099.

[10] Dumitriu, I. and Edelman, A. (2002). Matrix Models for Beta Ensembles. Journal of Mathematical Physics 43, 5830-5847.

[11] Dyson, F. J. (1962). The three fold way. Algebraic structure of symmetry groups and ensembles in quantum mechanics. J. Math. Phys. 3, 1199-1215.

[12] Eaton, M. (1983). Multivariate Statistics: A Vector Space Approach (Wiley Series in Probability and Statistics), John Wiley \& Sons Inc.

[13] Edelman, A. and Sutton, B. D. (2008). On the beta-Jacobi matrix model. Foundations of Computational Mathematics 8(2), 259-285.

[14] Forrester, P.J. (2006). Quantum conductance problems and the Jacobi ensemble. J. of Phys. A: Math. Gen. 39, 6861-6870.

[15] Jiang, T. (2005) Maxima of Entries of Haar Distributed Matrices, Probability Theory and Related Fields 131, 121-144.

[16] Jiang, T. (2006). How many entries of a typical orthogonal matrix can be approximated by independent normals? Ann. Probab. 34(4), 1497-1529.

[17] Jiang, T. (2009). Approximation of Haar distributed matrices and limiting distributions of eigenvalues of Jacobi ensembles. Probability Theory and Related Fields 144(1), 221-246. 
[18] Jalabert, R.A., Pichard, J.-L. and Beenakker, C.W.J. (1994). Universal Quantum Signatures of Chaos in Ballistic Transport. Europhysics Lett. 27, 255-260.

[19] Killip, R. (2008). Gaussian fluctuations for beta ensembles, available at "http://arxiv.org/abs/math/0703140".

[20] Killip, R. and Nenciu, I. (2004). Matrix models for circular ensembles. International Mathematics Research Notices 50, 2665-2701.

[21] Knapp, A. W. (2002). Lie Groups: Beyond An Introduction. Birkhauser Boston; 2nd ed.

[22] Kotz, S. Balakrishnan, N. and Johnson, N. L. (2000). Continuous Multivariate Distributions, Volume 1, Models and Applications, Wiley-Interscience; 2nd ed.

[23] Li, S. and Teng, C. (2001). Non-central quaternion $\chi^{2}, t$ and $F$ distributions and their properties. Journal of Mathematical Study 34(2), 170-173.

[24] Lida, S., Weidenmüller, M.A. and Zuk, J.A. (1990). Statistical scattering theory, the supersymmetry method and universal conductance fluctations. Ann. Phys. 200, 219-270.

[25] Lippert, R. A. (2003). A matrix model for the beta-Jacobi ensemble, Journal of Mathematical Physics 44(10), 4807-4816.

[26] Male, C., Le Caër, G. and Delannay, A. (2007). $1 / f^{\alpha}$ noise in the fluctuations of the spectra of tridiagonal random matrices from the $\beta$-Hermite ensemble. Physical Review E $76,042101$.

[27] Matsumoto, S. Averages of ratios of characteristic polynomials in circular beta-ensembles and super-Jack polynomials. Available at "http://arxiv.org/abs/0805.3573".

[28] Mehta, M. L. (1991). Random Matrices, 2nd Ed., Academic Press, Boston.

[29] Mezzadri, F. (2007). How to generate random matrices from the classical compact groups. Notices to the AMS 54(5), 592-604.

[30] Muirhead, R. J. (2005). Aspects of Multivariate Statistical Theory (Wiley Series in Probability and Statistics), Wiley-Interscience, 2nd Ed.

[31] Novaes, M. (2008). Statistics of quantum transport in chaotic cavities with broken timereversal symmetry. Phys. Rev. B 78, 035337.

[32] Relaño, A., Muñoz, L., Retamosa, J., Faleiro, E. and Molina, R. A. (2008). Powerspectrum characterization of the continuous Gaussian ensemble. Phys. Rev. E 77, 031103.

[33] Procesi, C. (2005). Lie Groups: An Approach through Invariants and Representations. Springer. 
[34] Savin, D. V. and Sommers, H. -J (2006). Shot nosies in chaotic cavities with an arbitrary number of open channels. Phy. Rew. B 73, 081307(R).

[35] Sutton, B. D. The Stochastic Operator Approach to Random Matrix Theory, PhD thesis at MIT, available at "http://faculty.rmc.edu/bsutton/".

[36] Teng, C. and Fang, K. (1997). Statistical analysis based on normal distribution of quaternion. Contemporary Multivariate Analysis and Its Applications, May 19-20, Hong Kong. 